\title{
Modelling of explosive events in the solar transition region in a $2 \mathrm{D}$ environment
}

\section{Various MHD experiments}

\author{
I. Roussev ${ }^{1,2}$, K. Galsgaard ${ }^{3}$, R. Erdélyi ${ }^{4}$, and J. G. Doyle ${ }^{2}$
}

1 High Altitude Observatory, NCAR, 3450 Mitchell Lane, Boulder, CO 80301, USA

e-mail: ilr@ucar.edu

2 Armagh Observatory, College Hill, Armagh, BT61 9DG, N. Ireland

e-mail: ilr@star.arm.ac.uk; jgd@star.arm.ac.uk

3 School of Mathematics and Statistics, University of St. Andrews, St Andrews, Fife KY16 9SS, Scotland e-mail: klaus@mcs.st-and.ac.uk

${ }^{4}$ Space \& Atmosphere Research Center, Dept. of Applied Mathematics, University of Sheffield, Hicks Building, Hounsfield Road, Sheffield, S3 7RH, England

Received 7 March 2001 / Accepted 28 May 2001

\begin{abstract}
We examine the response of various physical environments representing the solar atmosphere to a magnetic reconnection event. The reconnection is driven by a localized increase of the magnetic diffusivity in the current concentration formed between two magnetic fluxes of opposite polarity. The time dependent evolution is then followed by numerically solving the 2-dimensional (2D) dissipative magnetohydrodynamic (MHD) equations, including also effects of thermal conduction, radiative losses, and volumetric heating.

This work continues a previous related study (Roussev et al. 2001a), and compares results obtained from exploring different initial states. The choice of the initial states is found to be crucial to the dynamics of the reconnection jets. The numerical experiments are aimed at modelling transient events on the quiet Sun, with an emphasis on explosive events. The 2D reconnection experiments presented in this paper are the basis for a detailed analysis on the line synthesis in transition region resonant lines, presented by Roussev et al. (2001b).
\end{abstract}

Key words. MHD - Sun: atmosphere - Sun: chromosphere - Sun: UV radiation - Sun: magnetic fields

\section{Introduction}

There are remarkable observations in high resolution UV spectra taken by the High Resolution Telescope and Spectrograph (Brueckner \& Bartoe 1983; Dere et al. 1989). These reveal very small spatial regions in the solar atmosphere of sudden enhancements in the emission line intensities. Observed emission lines possess strongly broadened non-Gaussian, Doppler shifted profiles that involve spatial regions of 2 arcsec or less in size. Similar observations in mid-transition region lines of SMM UVSP (see, e.g., Porter et al. 1987) and more recently by SUMER (Innes et al. 1997; Perez et al. 1998, 1999; Erdélyi et al. 1997, 1998) and by TRACE (Erdélyi et al. 1999, 2001) have confirmed the existence of these very localized highvelocity events. Brueckner \& Bartoe (1983) classified these events as explosive events (also turbulent events), and reported that they could be detected only in emission lines

Send offprint requests to: R. Erdélyi,

e-mail: Robertus@sheffield.ac.uk formed between $2 \times 10^{4} \mathrm{~K}$ and $2 \times 10^{5} \mathrm{~K}$. Some recent observations taken by SUMER (Erdélyi et al. 1997, 1998; Innes et al. 1997; Perez et al. 1998, 1999) show explosive events at even higher temperatures up to $6.3 \times 10^{5} \mathrm{~K}$ (Ne VIII $770 \AA$ ).

Individual explosive events evolve on time scales ranging from 20 to $200 \mathrm{~s}$, with a lifetime histogram peaking at about $40 \mathrm{~s}$. Many of the events, however, exhibit quite complicated evolution and therefore it is not always possible to assign a single time scale for each individual event.

Nowadays, solar explosive events are believed to be a manifestation of magnetic reconnection on the Sun, taking place most probably when newly emerging flux interacts with the pre-existing magnetic field (Parker 1987; Spruit et al. 1987). Since explosive events are mainly observed at the edges of the cell boundaries in the "quiet" Sun, an ongoing reconnection must involve relatively small concentrations of magnetic flux. The process of magnetic reconnection usually occurs in response to changes in the spatial distribution of the magnetic flux. These changes 
may be due to a pure magnetic flux emergence as said before or/and foot point motions of existing flux. In the "quiet" Sun we expect that these processes often run in parallel. For the "quiet" Sun magnetic fields involved in the production of explosive events convection is probably the most important source of foot-point motions (Spruit et al. 1987). As to the dynamic consequences of magnetic reconnection in the photosphere driven by convective instabilities beneath, a recent study made by Takeuchi \& Shibata (2001) suggests formation of solar spicules. This phenomenon could therefore be another example of magnetic reconnection on the Sun.

It is also believed that the emission line broadenings observed during explosive events are due to bi-directional outflow jets located on either side of a magnetic cancellation site. Innes et al. (1997) examined the time variations and spatial structure of explosive events observed by SUMER, and concluded that these are consistent with the hypothesis of bi-directional plasma jets produced by magnetic reconnection.

Table 1. Normalization units.

\begin{tabular}{|lll|}
\hline Physical Quantity & Notation & Typical Value \\
\hline \hline Length & $L_{0}$ & $3 \times 10^{8}(\mathrm{~cm})$ \\
Velocity & $V_{A 0}$ & $1.293 \times 10^{7}\left(\mathrm{~cm} \mathrm{~s}^{-1}\right)$ \\
Time & $t_{A 0}$ & $2.320 \times 10^{1}(\mathrm{~s})$ \\
Density & $\rho_{0}$ & $5.019 \times 10^{-14}\left(\mathrm{~g} \mathrm{~cm}^{-3}\right)$ \\
Temperature & $T_{0}$ & $1 \times 10^{5}(\mathrm{~K})$ \\
Magnetic field & $B_{0}$ & $8(\mathrm{G})$ \\
Thermal & $\kappa_{0}$ & $1.23 \times 10^{-6}$ \\
conduction & & $\left(\mathrm{erg} \mathrm{s}^{-1} \mathrm{~cm}^{-1} \mathrm{~K}^{-1}\right)$ \\
\hline
\end{tabular}

Table 2. Listing of the parameter set used in the four different experiments.

\begin{tabular}{|lllll|}
\hline Parameter & Exp A1 & Exp A2 & Exp B1 & Exp B2 \\
\hline \hline$\epsilon$ & 7 & 7 & - & - \\
$\Delta \rho$ & 4.5 & 4.5 & 0 & 0 \\
$\theta$ & 0.6 & 0 & 0.6 & 0 \\
$y_{\mathrm{s}}^{*}$ & $0.4 y_{\max }^{*}$ & $0.4 y_{\max }^{*}$ & 0 & 0 \\
$y_{0}^{*}$ & $0.4 y_{\max }^{*}$ & $0.4 y_{\max }^{*}$ & 0 & 0 \\
$\gamma$ & 1.667 & 1.667 & 1.667 & 1.667 \\
$\beta$ & 0.164 & 0.164 & 0.164 & 0.164 \\
$\eta_{0}^{*}$ & $1 / 200$ & $1 / 200$ & $1 / 250$ & $1 / 250$ \\
$\alpha$ & 150 & 150 & 150 & 150 \\
$t_{\mathrm{ri}}^{*}$ & $5 / 100$ & $5 / 100$ & $5 / 100$ & $5 / 100$ \\
$t_{\mathrm{end}}^{*}$ & 5 & 5 & 5 & 5 \\
\hline
\end{tabular}

\subsection{Numerical modelling of explosive events}

To date, there are numerous and extensive studies of modelling phenomena related to active regions (such as solar flares, X-ray jets, etc.), involving magnetic reconnection models (see Yokoyama \& Shibata 1996; Forbes \& Priest 1984; Chen et al. 1999). In order to explain the eruptive phase of solar flares, it has been suggested that fast magnetic reconnection, i.e. reconnection that does not depend on the plasma resistivity, may be responsible for the violent energy release observed during these events (see Magara et al. 1996, 1997; Magara \& Shibata 1999). It was found that models of solar flares involving fast magnetic reconnection agree with, and physically confirm some recent X-ray observations taken by Yohkoh.

Magnetic reconnection depends critically on the magnetic field topology and on the thermal properties of the embedded plasma. Separate studies are therefore needed for the purposes of modelling transient events in the "quiet" Sun, and in particular explosive events. Although the spatial and time scales, as well as plasma densities and temperatures, involved in flare events are different from the ones representing the "quiet" Sun transition region, the general description of the magnetic reconnection itself is similar.

Recently, there has been significant progress made in the numerical modelling of explosive events in the case of 1D hydrodynamics, and especially in computing the observable consequences, such as line profiles. Sarro et al. (1999) simulated the evolution of UV emission line profiles (e.g., C IV), caused by small-scale energy depositions (nano-flares) in a 1D loop. The line synthesis was made under the assumption of non-equilibrium ionization. The latter is critical since the dynamic time scales during these events may be small compared to the ionization and recombination times (see Sarro et al. 1999). Similar idea has been used to model the long-standing problem of transition region line-shifts (Teriaca et al. 1999).

The interaction between newly emerging magnetic flux and pre-existing coronal field was modelled by Jin et al. (1996) and Innes \& Tóth (1999) using 2D MHD experiments. These numerical studies supported the idea that the blue- and red-shifted Doppler components observed during explosive events are due to bi-directional outflow jets produced by magnetic reconnection. The results obtained, however, though showing the development of the strongly Doppler shifted components, could not reproduce the typical pattern of transition region line profiles observed during explosive events. All these calculations were carried out assuming equilibrium ionization of the emitting plasma, though short time scale events like explosive events may not fulfill the requirements of such an assumption.

The present study is aimed at examining the response of various physical environments to a magnetic reconnection event representing the "quiet" solar atmosphere. Magnetic reconnection is initiated by a localized increase of the plasma resistivity in a $2 \mathrm{D}$ current concentration. The numerical modelling includes effects of the nonlinear anisotropic thermal conduction, radiative losses, volumetric heating and dissipative 2D MHD. This work is a continuation of a previous related study (Roussev et al. 2001a, hereafter referred to as Paper I), and presents the 
results obtained from exploring different physical situations. Both studies are dedicated to model transient events on the "quiet" Sun, and in particular explosive events.

\section{Physical description of the problem}

\subsection{Basic equations}

We consider the same model representing the physical environment of a transition region as the one described in Paper I. Let us briefly recall the key ingredients. We assume the solar atmosphere to be an ideal gas. The effect of gravity is neglected for the sake of simplicity ${ }^{1}$. The governing equations of a $2 \mathrm{D}$ dissipative, radiative MHD read

$\frac{D \rho}{D t}=-\rho \nabla \cdot \boldsymbol{U}$

$\rho \frac{D \boldsymbol{U}}{D t}=-\nabla P+\boldsymbol{j} \times \boldsymbol{B}-\nabla \cdot \hat{\tau}$

$\nabla \cdot \boldsymbol{B}=0$

$$
\frac{\partial \boldsymbol{B}}{\partial t}=\nabla \times(\boldsymbol{U} \times \boldsymbol{B})-\nabla \times\left[\left(\eta+\eta_{\mathrm{loc}}\right) \nabla \times \boldsymbol{B}\right],
$$

$$
\begin{aligned}
\frac{\partial e}{\partial t}= & -\nabla \cdot(e \boldsymbol{U})-P \nabla \cdot \boldsymbol{U}+Q_{\mathrm{visc}}+Q_{\text {Joule }} \\
& -\nabla \cdot \boldsymbol{q}-L_{\mathrm{r}}+S
\end{aligned}
$$

where $D / D t \equiv \partial / \partial t+\boldsymbol{U} \cdot \nabla$, and $\rho, P, \boldsymbol{U}, \boldsymbol{B}, \boldsymbol{j}, \eta, \hat{\tau}$, $e=P /(\gamma-1), Q_{\text {visc }}, Q_{\text {Joule }}, \boldsymbol{q}, S, L_{\mathrm{r}}$ are the mass density, kinetic gas pressure, velocity, magnetic field, electric current density, magnetic diffusion coefficient (numerical), viscous stress tensor (numerical), internal energy, viscous dissipation, Joule dissipation, heat flux, volumetric heating rate, and radiative losses, respectively. By $\eta_{\text {loc }}$ we denote an ad-hoc coefficient of localized magnetic diffusion used in our experiments to initiate magnetic reconnection. Also, $\boldsymbol{j}, P, \boldsymbol{q}$, and $L_{\mathrm{r}}$, are explicitly given by

$\boldsymbol{j}=\nabla \times \boldsymbol{B}, P=\rho T$,

$\boldsymbol{q}=-\kappa_{\|} \nabla_{\|} T$, and $L_{\mathrm{r}}=n_{\mathrm{e}} n_{\mathrm{p}} Q(T)$,

where $\kappa_{\|}$is the thermal conduction along the magnetic field lines given by $\kappa_{\|}=\kappa_{0} T^{5 / 2}$ (subscript $\|$ in the nabla operator refers to values along the magnetic field). Here $n_{\mathrm{e}}$ and $n_{\mathrm{p}}$ are the electron and proton number densities, respectively. We work in units where the gas constant, $R_{0}$, is equal to the mean molecular weight, $\mu$, and the magnetic permeability is set to unity. For the radiative loss function, $Q(T)$, we have chosen the form given by Cook et al. (1989) calculated assuming photospheric abundances.

\footnotetext{
${ }^{1}$ We do not try to model solar transition region, either in conductively heated or in cool loop configurations. Neglect of gravity means that, though flux tubes have shallow temperature gradients and so are more like cool loops, they are not stratified. This we expect to influence our results (see Sect. 4.2).
}

\subsection{Initial state}

We also skip the detailed description of the initial state, and give only the necessary final formulae and relations as derived for dimensionless quantities and parameters of the problem (for details see Paper I).

In terms of dimensionless physical quantities and model parameters, the initial state reads

$\boldsymbol{u}=0$,

$b_{x}=0, b_{y}=\tanh \left(25 x^{*}\right)$,

$e^{*}=\frac{1}{2(\gamma-1)}\left(1+\beta-b_{y}^{2}\right)$,

$$
\begin{aligned}
\rho^{*} & =\left[\frac{\left(1+\beta-b_{y}^{2}\right)}{\beta}\right]^{\theta} \\
& \times\left\{1+\Delta \rho\left\{1-\tanh \left[\epsilon\left(y^{*}+y_{\mathrm{s}}^{*}\right)\right]\right\}\right\},
\end{aligned}
$$

and

$T^{*}=(\gamma-1) \frac{e^{*}}{\rho^{*}}$,

where $\gamma, \beta, \epsilon, \theta, \Delta \rho$ are the adiabatic index, plasma $\beta$ parameter, steepness of the transition region, free parameter (one over the polytropic index) which controls the density profile across the current concentration (i.e. in the $x$-direction), and a parameter $\Delta \rho$ which defines the density (and also temperature) jump across the transition region, i.e. $2 \Delta \rho+1$, respectively.

We consider a numerical domain of size $\left[2 x_{\max }^{*}, 2 y_{\max }^{*}\right]$, where $-x_{\max }^{*} \leq x^{*} \leq x_{\max }^{*}$, $-y_{\max }^{*} \leq y^{*} \leq y_{\max }^{*}$.

A further assumption in this 2D model is an energy balance for the initial state (for more details see Paper I) $S^{*}-\nabla^{*} \cdot \boldsymbol{q}^{*}-L_{r}^{*}=0 ;$ at $t^{*}=0$.

The normalization units adopted in this study and used in the different experiments we carry out are given in Table 1 . Note these values are referred to the high temperature region far away from the current concentration, and are defined at the location with coordinates $\left(x^{*}, y^{*}\right)=\left(-x_{\max }^{*}, y_{\max }^{*}\right)$.

In order to initiate a magnetic reconnection event in the initially equilibrium environment, we induce a localized magnetic diffusion for a finite time. The explicit form of the assumed diffusivity, $\eta_{\text {loc }}^{*}$, is

$$
\begin{aligned}
\eta_{\mathrm{loc}}^{*}\left(x^{*}, y^{*} ; t^{*}\right)= & \eta_{0}^{*} F^{*}\left(t^{*}\right) \\
& \times \exp \left\{-\alpha\left[x^{* 2}+\left(y^{*}+y_{0}^{*}\right)^{2}\right]\right\},
\end{aligned}
$$

where

$F^{*}\left(t^{*}\right)= \begin{cases}\frac{t^{*}}{t_{\mathrm{ri}}^{*}}, & 0 \leq t^{*} \leq t_{\mathrm{ri}}^{*} \\ 1, & t_{\mathrm{ri}}^{*}<t^{*} \leq t_{\mathrm{end}}^{*} \\ 0, & t_{\mathrm{end}}^{*}<t^{*}\end{cases}$

Here $\eta_{0}^{*}$ is the normalized value of the magnetic diffusion coefficient defined as the reciprocal of a characteristic magnetic Reynolds number, $R_{\mathrm{m}}$, in the diffusion region (i.e. $\left.\eta_{0}^{*}=1 / R_{\mathrm{m}}\right)$. Note that $\eta_{0}^{*}$ is held constant throughout each numerical experiment (refer to Table 2). 

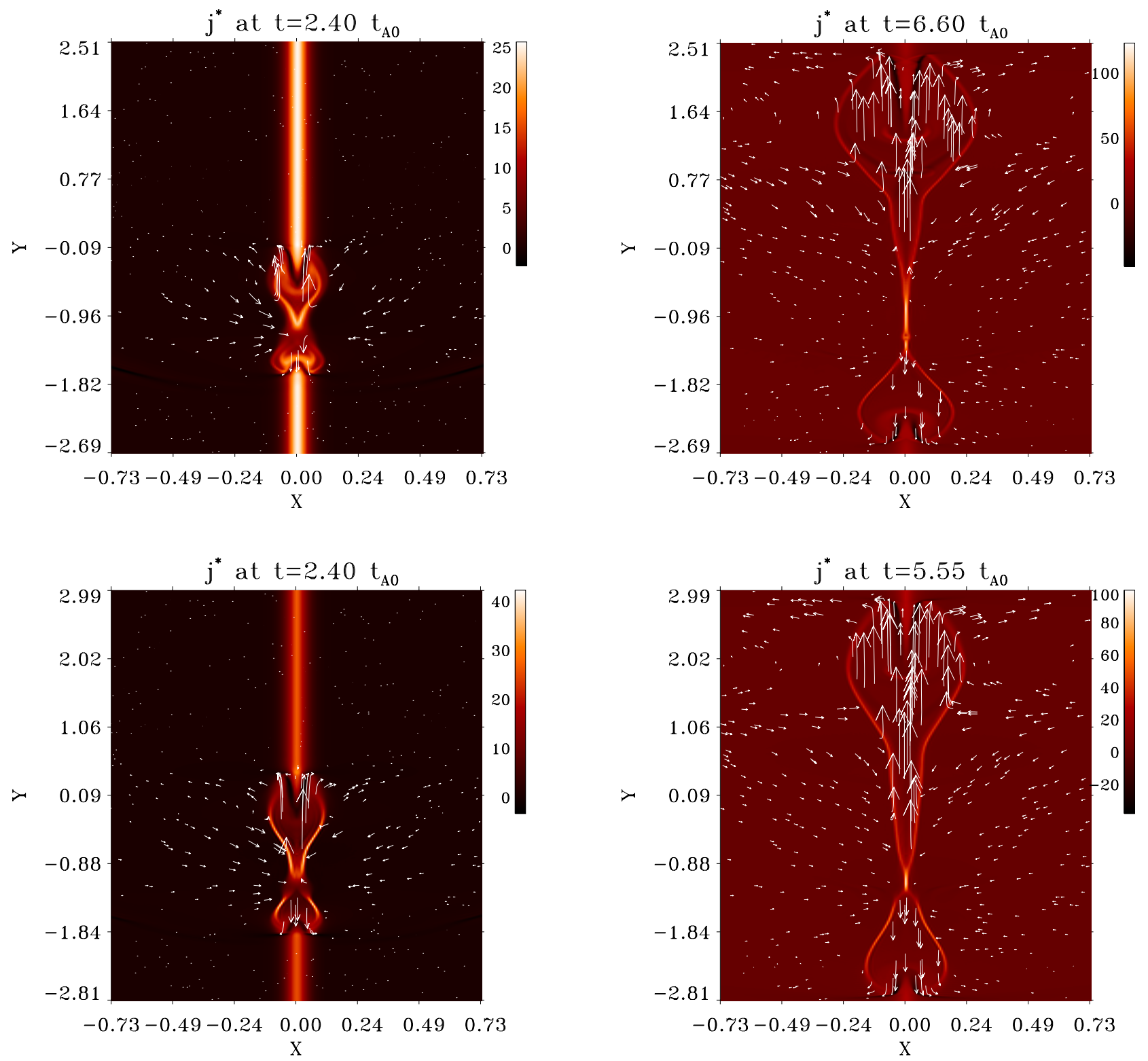

Fig. 1. Images of the $z$-component of the current, $j^{*}$, are shown at two given times (as indicated on each plot) for the two experiments "A". The top panels show experiment "A1", while the bottom ones refer to experiment "A2", respectively. Short streak lines represent the velocity field at given time (see text for more details). The maximum flow velocity is of magnitude, as follows: 0.37 (top left), 1.01 (top right), 0.64 (bottom left), and 1.02 (bottom right), respectively.

\subsection{Set of experiments}

Based on the 2D model presented above, we explore the response of four physical environments to a magnetic reconnection event, initially each of them in equilibrium. The following cases are considered in particular:

- Exp A1: a transition region and a density enhancement in the current "sheet" $(\Delta \rho>0, \theta>0)$;

- Exp A2: a transition region and a uniform mass density across the current "sheet" $(\Delta \rho>0, \theta=0)$;

- Exp B1: no transition region with a density enhancement in the current "sheet" $(\Delta \rho=0, \theta>0)$;

- Exp B2: no transition region with a uniform mass density across the current "sheet" $(\Delta \rho=0, \theta=0)$.
Table 2 gives the parameter sets chosen for the four different experiments. Experiments "A" may represent the case when two magnetic flux tubes of opposite polarity are rooted in the photosphere and advected into contact due to, for example, their foot-point motions. Experiments "B", in turn, may refer to the case when an emerging magnetic flux tube interacts with a preexisting horizontal magnetic field of opposite polarity. Furthermore, the setup for experiments "B" could also represent case "A", if "cool" flux tubes are considered. In this case there is no temperature stratification along the magnetic field lines.

In all these experiments, the initiation of a non-zero localized magnetic diffusivity, $\eta_{0}^{*}$, drives annihilation of the 

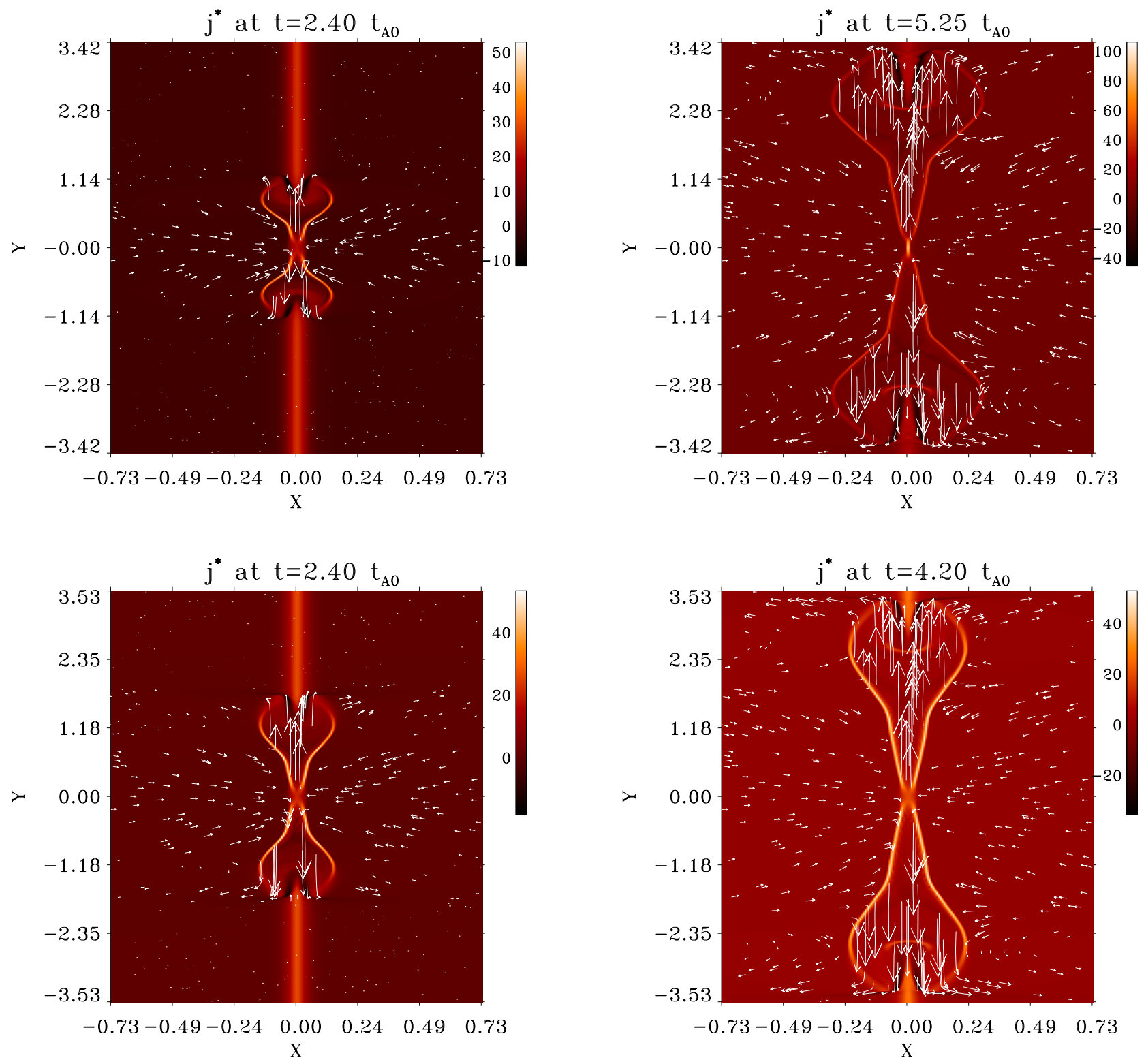

Fig. 2. Images of the $z$-component of the current, $j^{*}$, are now shown at two given times for the two experiments "B". The top panels show experiment "B1", while the bottom ones refer to experiment "B2", respectively. Short streak lines represent the velocity field at given time. The maximum flow velocity is of magnitude, as follows: 0.80 (top left), 1.01 (top right), 0.89 (bottom left), and 0.99 (bottom right), respectively.

two oppositely directed magnetic fluxes, and thus initiates an X-type magnetic reconnection event.

\section{Numerical method}

All numerical experiments presented above are performed using a 2D compressible MHD code based on staggered meshes (see Paper I). The MHD equations are solved in a $2 \mathrm{D}$ domain using a stretched grid in the $x$-direction in order to better resolve the region around the current concentration at which the most important dynamics of the experiments takes place. Furthermore, the applied stretching of the grid "moves" the $x$-boundaries far enough away in order not to influence the solution inside the domain.
In the $y$-direction, however, there is no stretching applied since high resolution is needed at every point. The grid-size used in the four experiments is $N_{x} \times N_{y}=$ $400 \times 700$, where $50 \%$ of the grid points in the $x$-direction are located within $-0.7 \leq x^{*} \leq 0.7$ (which is 0.23 times the size of the domain in the $x$-direction). The smallest grid spacing across the current concentration (in the $x$-direction) is assigned at $x^{*}=0$, and corresponds to $\left(\Delta x^{*}\right)_{\min }=6.05 \times 10^{-3}$. The one at the $x$-boundaries is longer than this because of the stretching applied, and is equal to $\left(\Delta x^{*}\right)_{\max }=7.05 \times 10^{-2}$. In contrast, the grid spacing in the $y$-direction is uniform, and $\Delta y^{*}=8.67 \times 10^{-3}$.

The MHD code used in our experiments conserves mass, momentum, energy, and magnetic field divergence. 

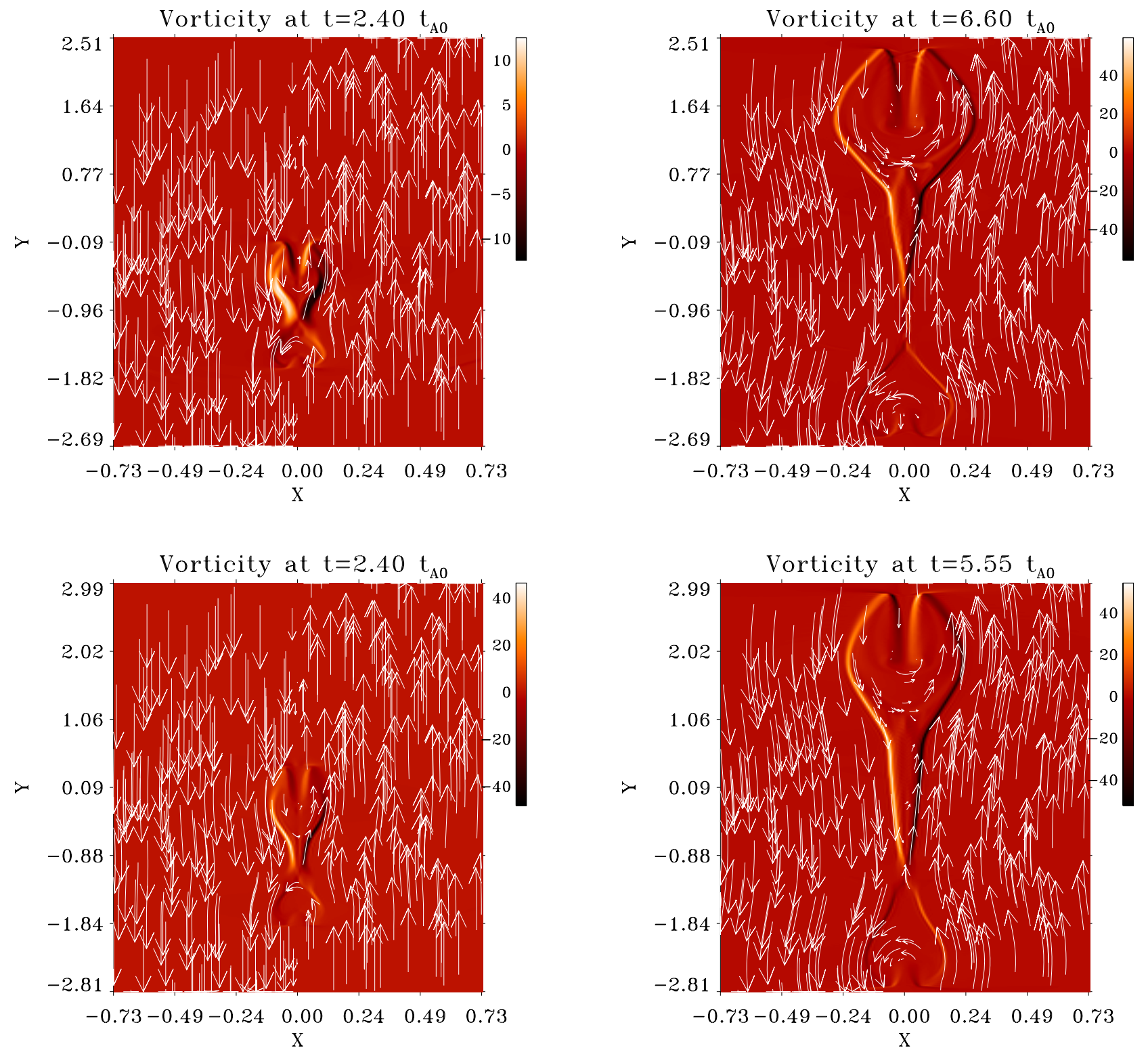

Fig. 3. Vorticity of the velocity field is shown at two given times (as indicated on each plot) for experiment "A1" (top) and "A2" (bottom), respectively. Short field lines are plotted to show the magnetic field topology at the given time (see text for details). The maximum value of the magnitude of this field is as follows: 1.15 (top left), 1.23 (top right), 1.15 (bottom left), and 1.19 (bottom right), respectively.

In order to derive the partial derivatives in the MHD equations, a sixth-order method is applied. The interpolation of various functions between the grid points is carried out using a fifth-order method. The numerical viscosity and magnetic diffusivity are both handled using a combined second and fourth-order method extended with a discontinuous capture mechanism. The solution is advanced in time using a third-order predictor-corrector method. The boundaries are, for simplicity, taken to be ideally conducting and impenetrable. The parallel velocity at the boundaries is kept as zero throughout the experiments.

\section{Discussion on the numerical results}

This section summarizes the results of our numerical modelling. The common dynamics of the four experi- ments is discussed in Sect. 4.1. A comparison between the two "A" experiments is made in Sect. 4.2. Similarly, experiments "B" are discussed in Sect. 4.3, and final conclusions are drawn in Sect. 5.

\subsection{Common dynamic properties}

The "A" experiments are carried out in a numerical domain of size $\left[2 x_{\max }^{*}, 2 y_{\max }^{*}\right]=[6,6]$, while the "B" experiments are performed in a domain of size $[6,8]$. The four experiments evolve on different dynamical time scales, and therefore end at different times. The end times are respectively: $t_{\mathrm{end}}^{* \mathrm{~A} 1}=6.60$ for experiment "A1"; $t_{\mathrm{end}}^{* \mathrm{~A} 2}=5.55$ for experiment "A2"; $t_{\text {end }}^{* \mathrm{~B} 1}=5.25$ for experiment "B1"; and $t_{\mathrm{end}}^{* \mathrm{~B} 2}=4.20$ for experiment "B2". The localized magnetic 

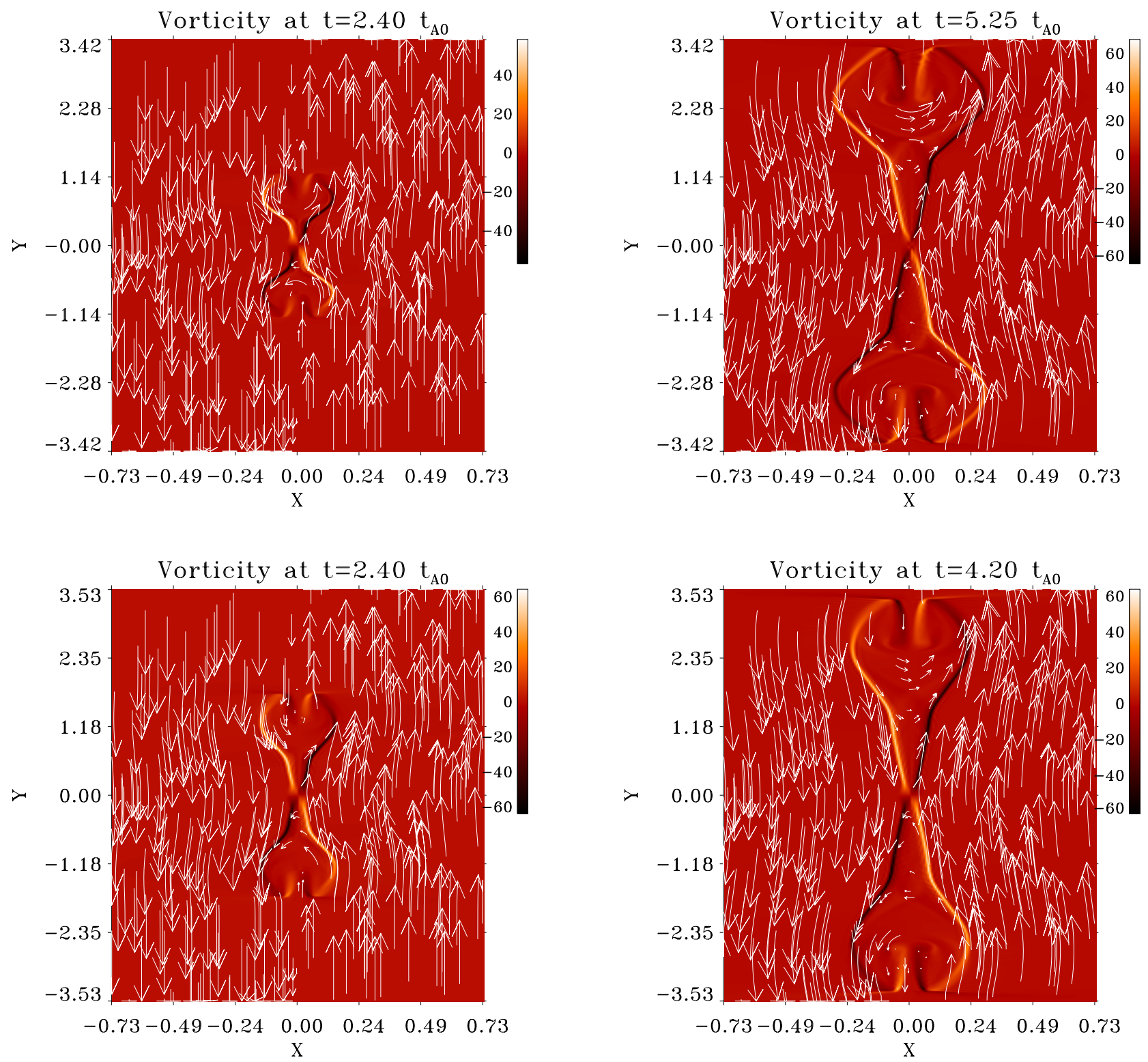

Fig. 4. Vorticity of the velocity field at two given times is now shown for experiment "B1" (top) and "B2" (bottom), respectively. Short field lines represent the magnetic field topology at given time. The maximum value of the magnitude of this field is as follows: 1.23 (top left), 1.21 (top right), 1.16 (bottom left), and 1.15 (bottom right), respectively.

diffusion term is switched on at time $t^{*}=0$, and is maintained until time $t^{*}=5.0$. All times are given in units of $t_{A 0}$ (see Table 1 ).

In order to ease the following analysis of the general dynamics of the four experiments, we further ignore the net effect of the radiative losses, thermal conduction and volumetric heating to the corresponding MHD solutions as being small compared to the importance of the other physics involved. The impact of these processes to the MHD calculations is discussed at the end of this section in Sect. 4.1.1.

Once the localized magnetic diffusion is induced, the initial force balance is destroyed, and the $2 \mathrm{D}$ current concentration evolves into a $2 \mathrm{D} \mathrm{X}$-point topology centered in the localized magnetic diffusion patch. Since the newly reconnected field lines are extremely curved they possess large tension force in the $\pm y$-direction. Therefore, plasma from the diffusion region is pulled out by the reconnected magnetic field, and is accelerated along the current concentration in the $y$-directions. As a dynamic reaction to the decrease in the plasma and magnetic pressure in the diffusion region, an inflow of magnetized plasma towards the $\mathrm{X}$-point is initiated. This inflow advects new magnetic flux into the diffusion region, and as the reconnection proceeds, the outflow jets continue to propagate along in the $\pm y$-direction in the current "sheet". As the localized magnetic diffusion is maintained constant from $t_{\mathrm{ri}}^{*}$ until $t_{\mathrm{end}}^{*}$, a steady state magnetic reconnection situation is eventually approached. Since the speed of a slow MHD mode is zero perpendicular to the magnetic field, no matter how slow the inflow of material from the outside regions is, it will always be supersonic with respect to the 

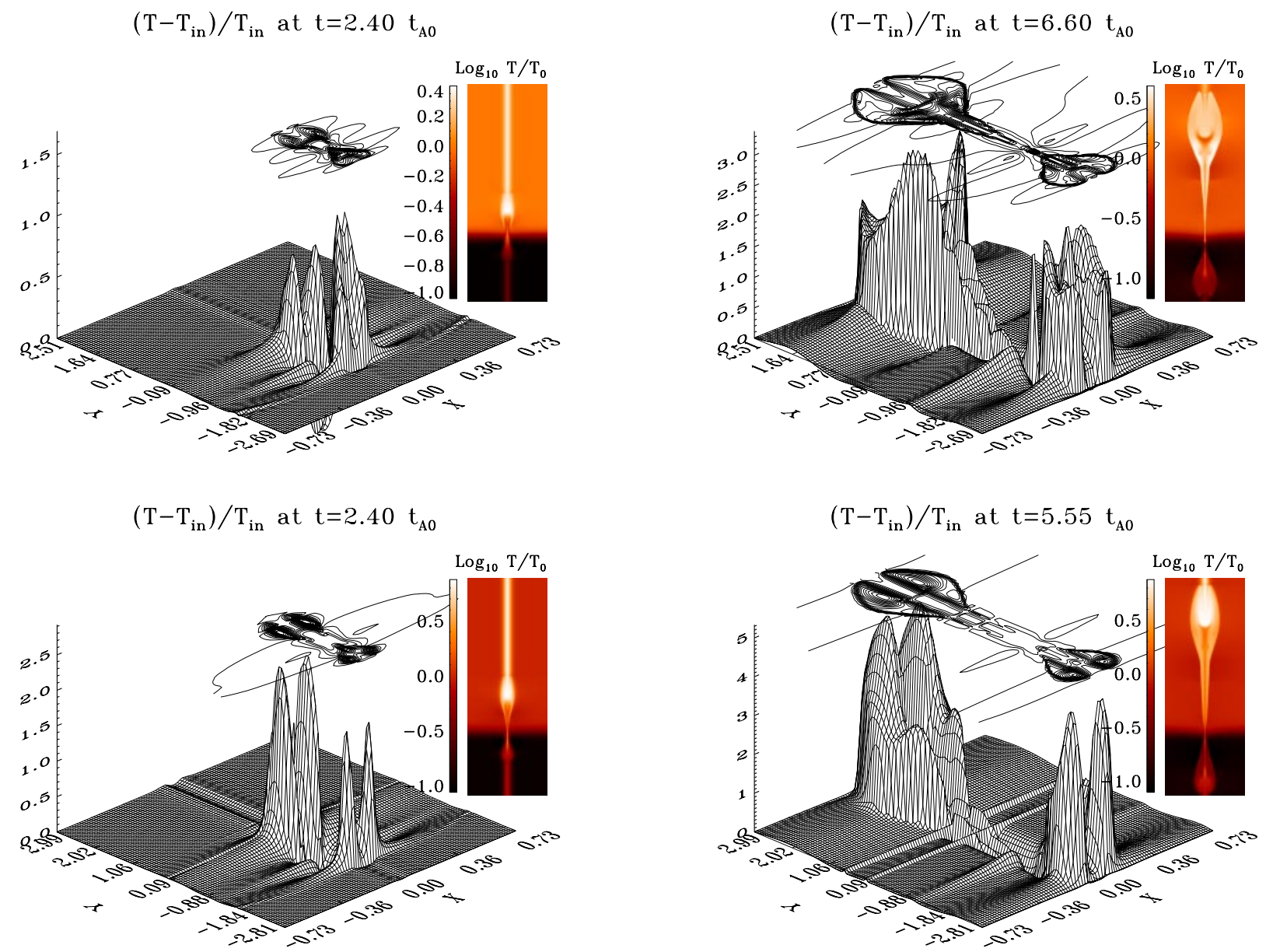

Fig. 5. Relative changes in the temperature, $\left(T-T_{\mathrm{in}}\right) / T_{\mathrm{in}}$, with respect to the initial temperature field, $T_{\mathrm{in}}$, are shown for the two experiments "A" at the same given times as in Fig. 1. The top panels show experiment "A1", while the bottom ones refer to experiment "A2", respectively. Images at the top right panel of each figure shows the logarithm of the temperature, $\log _{10}\left(T / T_{0}\right)$, at given time (see text for more details). Note also that $T_{0}$ is normalization value of the temperature, while $T_{\mathrm{in}}$ is the initial temperature field in the physical domain.

slow MHD mode. Slow MHD shocks are therefore formed at the locations where inflows are initiated. Because of the steep gradient of the kinetic gas pressure across the slow MHD shocks, force balance is reached at some high enough value of the electric current density, $j^{*}$. The positions of the slow shocks can be therefore traced by the locations of strong electric current, $j^{*}$, around the jets. These shock regions can be seen in Figs. 1-2, where the background shading in the figures represents the current density. Figure 1 refers to the two experiments "A", while Fig. 2 represents experiments "B", respectively. In order to better show the fine structure of the current concentration, we plot only the $-0.73 \leq x^{*} \leq 0.73$ part of the domain in the $x$-direction. The plot range in the $y$-direction is larger than the one in the $x$-direction, and is chosen to show the two jets in the plotted domain towards the end of each experiment. The velocity field is plotted as short streak lines. Note this field is symmetric with respect to $x^{*}=0$, though the streak lines are traced from randomly chosen starting points in the plot domain. The flow pattern is found to have slightly converging shape in the vicinity of the X-point, and the reconnection event would there- fore, in a steady state, be classified as a Petschek type according to Priest \& Forbes (1986).

Once the localized magnetic diffusion is switched off $\left(t=t_{\text {end }}^{*}\right)$, the kinetic gas pressure at the X-point starts increasing, and therefore force balance is eventually reached closer to the symmetry axis, $x^{*}=0$, at increased values of the electric current density. Note the narrow regions of strong current along the symmetry axis $x^{*}=0$ in Fig. 1 (right column) and Fig. 2 (top right panel).

The corresponding changes in the magnetic field topology are shown in Fig. 3 for the two "A" experiments and in Fig. 4 for the two "B" experiments, respectively. In both figures the magnetic field is plotted as short field line traces, whereas the background images show the vorticity of the velocity field. Note again that the magnetic field is symmetric with respect to $x^{*}=0$, but that the positions of the short field line traces are randomly selected. The vorticity in 2D magnetic reconnection experiments has a characteristic quadrupolar structure, clearly indicating the locations of the X-point (zero vorticity) and slow mode shocks (high absolute value of the vorticity). 

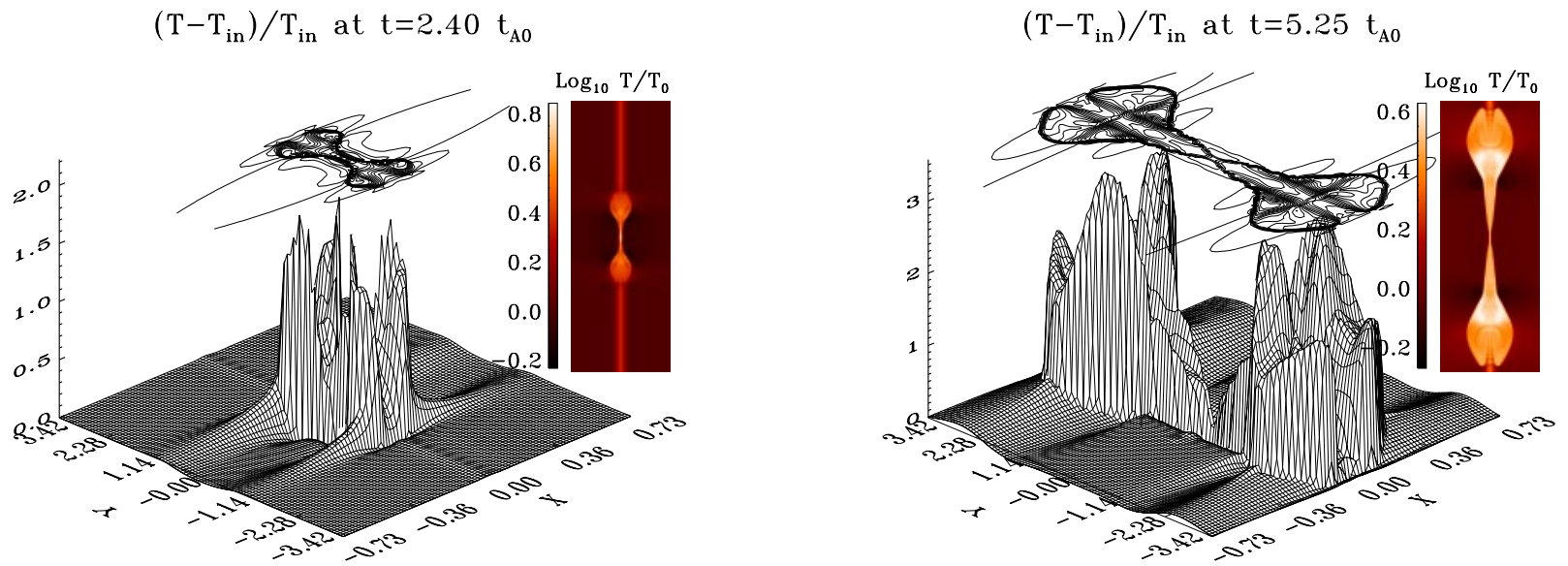

$$
\left(\mathrm{T}-\mathrm{T}_{\text {in }}\right) / \mathrm{T}_{\text {in }} \text { at } \mathrm{t}=2.40 \mathrm{t}_{\mathrm{A} 0}
$$
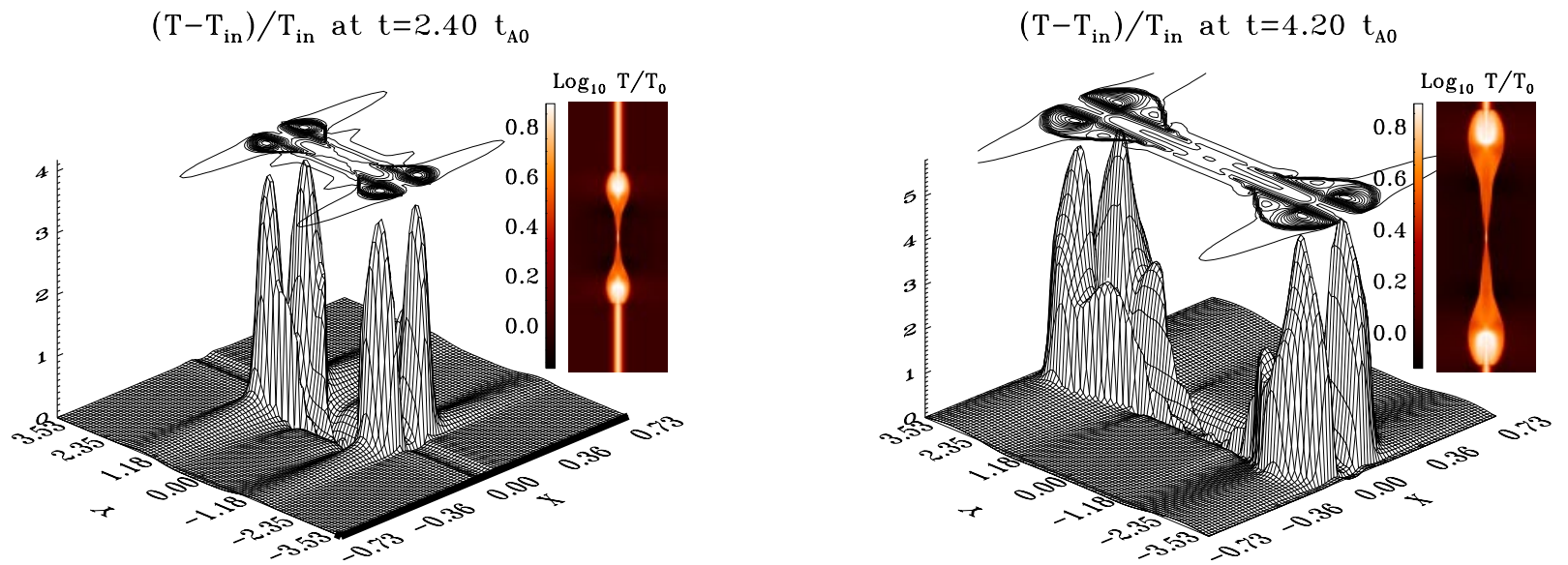

Fig. 6. Relative changes in the temperature, $\left(T-T_{\mathrm{in}}\right) / T_{\mathrm{in}}$, with respect to the initial temperature field, $T_{\mathrm{in}}$, are now shown for the two experiments "B" at the same given times as in Fig. 2. The top panels refer to experiment "B1", whereas the bottom ones show experiment "B2", respectively. Images at the top right panel of each figure shows the logarithm of the temperature, $\log _{10}\left(T / T_{0}\right)$, at given time.

Note that all the plots in the left column of Figs. 1-8 represent one and the same instant time of the four experiments, $t^{*}=2.40$, while the plots in the right column refer to the last snapshot taken in each individual experiment.

Since the initial magnetic field is uniform along the current concentration, as well as the current density is constant along a particular field line, the Lorentz forces accelerating the outflow jets are of the same magnitude. As a result, the value of the plasma density in the current "sheet" determines the relative acceleration of these jets reached in the four different experiments. Hence, the lower the mass density in the current concentration (low value of $\theta$ ), the faster the evolution of the two jets. Consequently, the reconnection jets of experiments " $\mathrm{A}(\mathrm{B}) 2$ " evolve on shorter dynamic time-scales than the ones in experiments "A(B)1".

As the outflow jets develop, their thermal energy increases due to the conversion of kinetic energy into heat through shock heating and plasma compression. In turn, the thermal energy at the X-point decreases with time as a dynamic consequence of the rapid expansion the plasma experiences as it is advected away with the jets. Due to the fast expansion of the hot jets, the non-heated plasma surrounding the current concentration is pushed away. Furthermore, since the plasma $\beta$ outside the current concentration is less than unity (i.e., $\beta=0.164$ ), the increased kinetic gas pressure in the two jets needs to be balanced only by a small perturbation of the magnetic field (in the direction perpendicular to the current concentration). Thus, the expansion of the outflow jets in the $x$-direction is much smaller than the one in the $y$-direction. As a consequence of the time dependent increase of the thermal energy in the two jets, the new pressure balance is reached farther away from the symmetry axis $x^{*}=0$, as these jets propagate along the current "sheet". The locations of the slow shocks therefore also move away from the symmetry axis, as the distance to the $\mathrm{X}$-point along this axis is increased. Figures 1-4 show, the slow MHD shocks trace the external, tulip-like envelope of each jet. Note also the reconnection jets are fed not only by the diffusion region in the vicinity of the $\mathrm{X}$-point, but also by the standing slow shocks all the way through (see the velocity flow pattern around the slow shocks in Figs. 1 and 2).

The reconnection jets of each experiment reach supersonic and super Alfvénic velocities along the symmetry axis $x^{*}=0\left(V_{\mathrm{Alfv}}=0 ; C_{\mathrm{s}}(x)=\max \left(C_{\mathrm{s}}\right)\right.$, since 

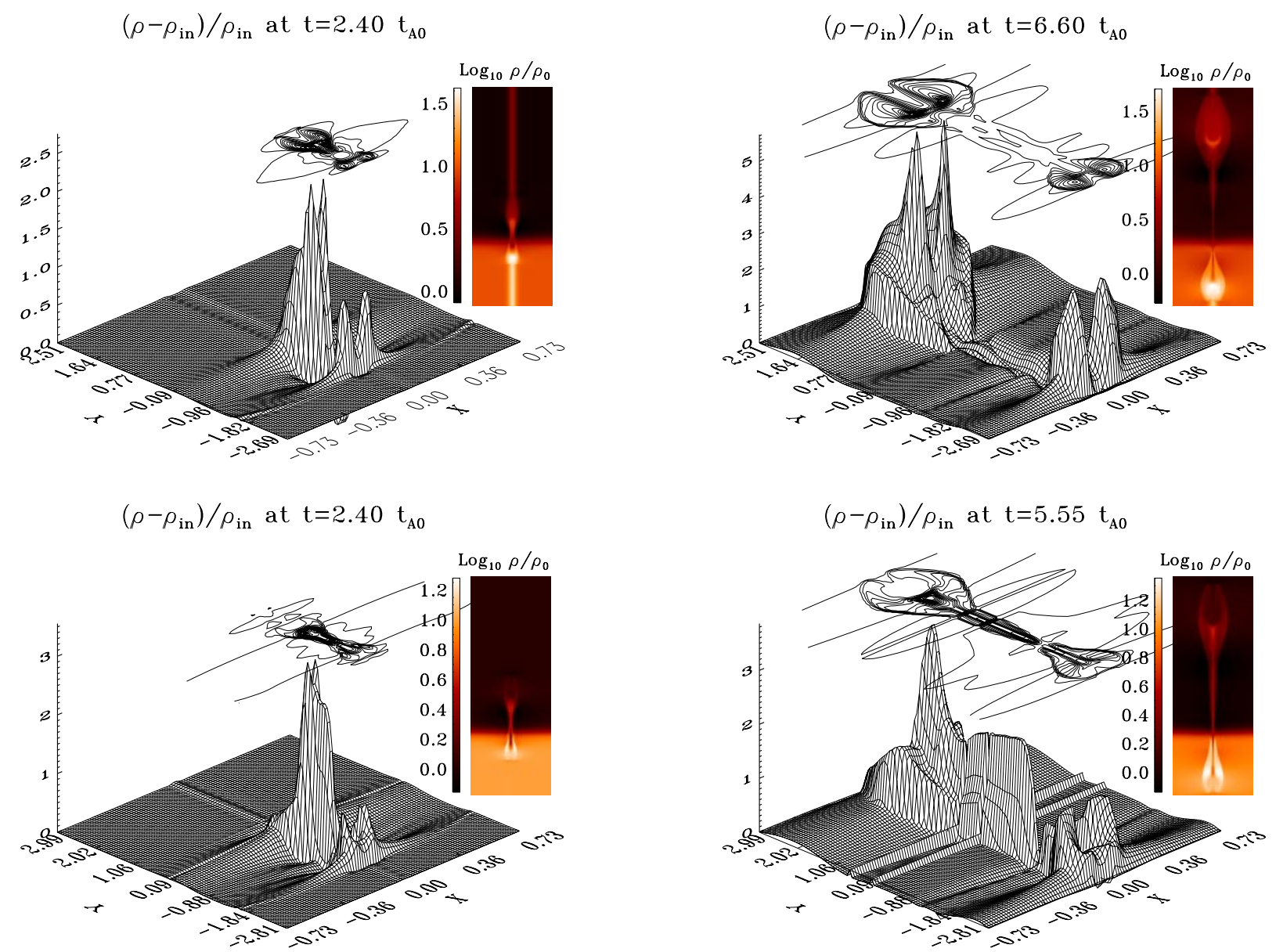

Fig. 7. Relative changes in the mass density, $\left(\rho-\rho_{\text {in }}\right) / \rho_{\text {in }}$, with respect to the initial density distribution, $\rho_{\text {in }}$, are shown for the two experiments "A" at two given times (as indicated on each figure). The top panels show experiment "A1", whereas the bottom ones refer to experiment "A2", respectively. Images at the top right panel of each figure shows the logarithm of the mass density, $\log _{10}\left(\rho / \rho_{0}\right)$, at given time (see text for more details). Note also that $\rho_{0}$ is normalization value of the mass density, while $\rho_{\text {in }}$ is the initial mass density distribution in the physical domain.

$T(x)=\max$ ), and bow shocks are consequently formed in front of each jet. As the distance to the symmetry axis in the $x$-direction is increased, the sound speed decreases, while the Alfvén speed increases in the initial background plasma. The bow shocks therefore become less steep, and at a given distance on both sides of the symmetry axis, $x^{*}=0$, the outflow jets will only be supersonic (and subAlfvénic). The lower the value of $\theta$ (i.e. the bigger the inhomogeneity of the plasma temperature across the current "sheet"), the steeper the gradient of both the sound speed, $C_{\mathrm{s}}$, and the Alfvén velocity, $V_{\mathrm{Alfv}}$, across the current concentration becomes. Furthermore, the lower the value of $\theta$, the more cusp-like the shape of the bow shocks is, as well as the more confined the structure of the outflow jets across the current "sheet" becomes. (Note that the lower the $\theta$ value, the closer to the symmetry axis $x^{*}=0$ the bow shocks become supersonic and sub-Alfvénic.)

The temperature and density structure of the reconnection jets, derived at the same snapshot as in Figs. 14, are shown in Figs. 5, 6 and Figs. 7, 8, respectively. Figures 5 and 7 refer to experiments "A", while Figs. 6 and 8 represent the two "B" experiments. Figures 5 and 6 show the relative changes in the temperature $\left(T-T_{\text {in }}\right) / T_{\text {in }}$, with respect to the initial distribution of the temperature, $T_{\mathrm{in}}$. On the surface and contour plots the domain is compressed in the $y$-direction and a square plot domain is shown. The adjacent panels, however, show the logarithm of the temperature, $\log _{10}\left(T / T_{0}\right)$, in the correct aspect ratio between the $x-$ and $y$-axes. In a similar manner, we present plots for the mass density, and Figs. 7, 8 show the relative changes in the mass density, $\left(\rho-\rho_{\text {in }}\right) / \rho_{\text {in }}$, with respect to the initial mass density distribution, $\rho_{\text {in }}$. Here the adjacent panels present the logarithm of the mass density, $\log _{10}\left(\rho / \rho_{0}\right)$. The surface and contour plots in Figs. 5-8 clearly show a distinct double-peak structure of each outflow jet in the relative changes, while the image plots of the temperature and mass density reveal single peaks. This difference is due to the initial profile of enhanced temperature (and enhanced mass density in experiments "A1" and "B1") in the current concentration, as well as because of the increasing width of the jet fronts as they expand with time in both the $x$ - and $y$-directions. (See also the 

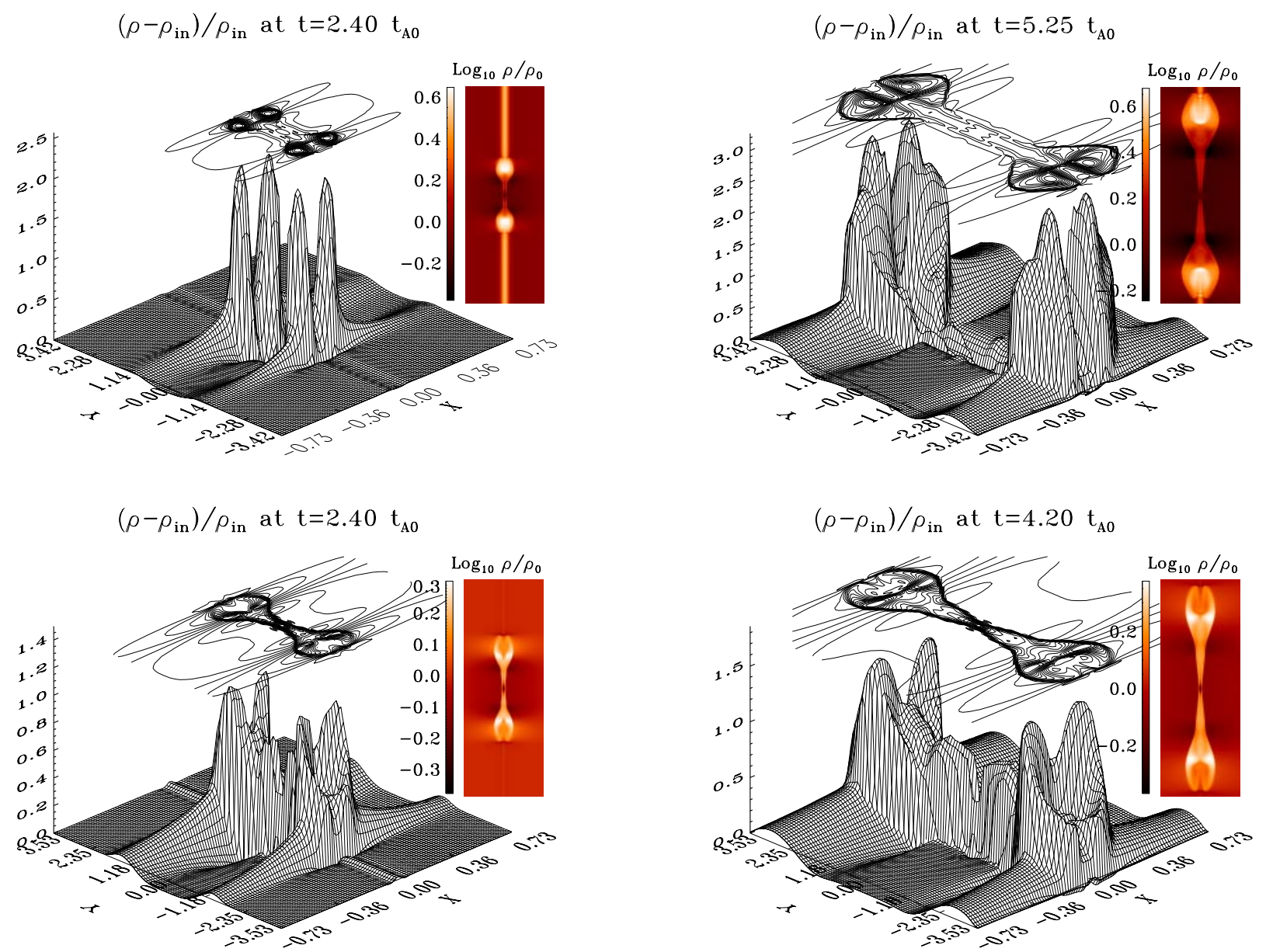

Fig. 8. Relative changes in the mass density, $\left(\rho-\rho_{\text {in }}\right) / \rho_{\text {in }}$, with respect to the initial density distribution, $\rho_{\text {in }}$, are now shown for the two experiments "B" at two given times. The top panels show experiment "B1", while the bottom ones refer to experiment "B2", respectively. Images at the top right panel of each figure shows the logarithm of the mass $\operatorname{density,~} \log _{10}\left(\rho / \rho_{0}\right)$, at given time.

discussion above regarding the profiles of the sound speed, $C_{\mathrm{s}}$, and the Alfvén velocity, $V_{\mathrm{Alfv}}$, across the current concentration for different values of the $\theta$ parameter.)

\subsubsection{Role of radiative losses, thermal conduction and volumetric heating}

In the analysis made so far, the effects of the thermal conduction, radiative losses and the volumetric heating were neglected for the sake of simplicity. The net effect of these two processes combined with the volumetric heating is quite complex and difficult to analyze due to the complicated temporal and spatial changes in mass density and temperature along the reconnection jets.

In the following, a quantitative analysis is made to address the importance of radiation, heat conduction and background heating on the overall physical picture of the numerical experiments. To do this, let us first define a characteristic dynamic time-scale on which changes in the thermal energy take place by, $t_{\mathrm{th}}^{*}=\left|\partial \ln e^{*} / \partial t^{*}\right|^{-1}$. The minimum value of $t_{\mathrm{th}}^{*}$ taken over the numerical domain at any given time indicates what is the shortest dynamical time scale on which the thermal energy changes relative to the Alfvén travel time, $t_{A 0}$. Once derived for all four experiments, it is found that the minimum value of $t_{\mathrm{th}}^{*}$ monotonically decreases in time. The fastest happens to be the evolution relevant to the particular setup of experiment "B2", while the slowest one is that of experiment "A1". The utmost minimum value of $t_{\mathrm{th}}^{*}$ is reached in all experiments towards their termination, and is of the order of 0.02. For a comparison, the time stepping controlled by the CFL condition at this phase of the experiments is about 10000 times smaller, and is controlled by the viscous dissipation.

In order to assess the importance of the radiative losses (and the relevant contribution to the volumetric heating) in the dynamics of magnetic reconnection examined here, we define a characteristic dynamic time-scale on which these operate by, $\Theta^{*}=\left|\left(L_{\mathrm{r}}^{*}-\left.L_{\mathrm{r}}^{*}\right|_{t^{*}=0}\right) / e^{*}\right|^{-1}$, where $\left.L_{\mathrm{r}}^{*}\right|_{t^{*}=0}$ are the radiative losses at time zero. By investigating the ratio of $\Theta^{*} / t_{\mathrm{th}}^{*}$, it is found that, on average, the radiative losses operate on a time-scale that is, respectively, 20 (A1), 40 (A2), 130 (B1), and 240 (B2) times longer than $t_{\mathrm{th}}^{*}$. There are, however, particular locations where $\Theta^{*} / t_{\mathrm{th}}^{*} \sim 1$. This occurs in the ad-hoc diffusion region (even after the localized diffusion is turned off), and 

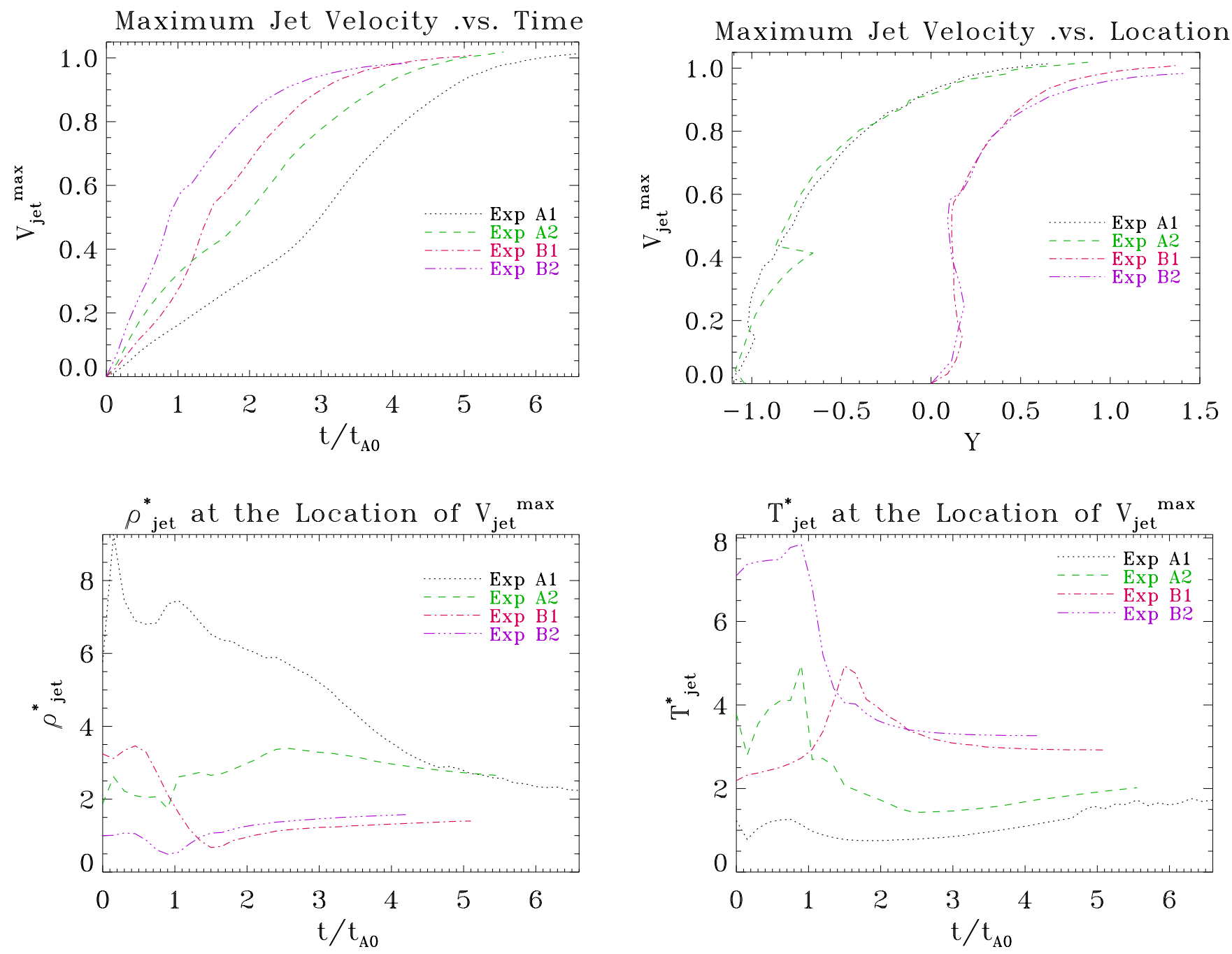

Fig. 9. Set of plots showing various physical quantities at the location of maximum jet velocity, $V_{\text {jet }}^{\max }$ (see text for details).

in the heads of "red" jets relevant to experiments "A1" and "A2". In the former region, it is found that the adhoc background heating, $\left.L_{\mathrm{r}}^{*}\right|_{t^{*}=0}$, dominates the radiative time-scale defined above, while in the "red" jets it is the radiative losses which do so.

In a similar manner, the dynamical time-scale of the heat conduction (and the relevant contribution to the volumetric heating $)$ is defined by: $\Xi^{*}=\mid\left[\left(\nabla^{*} \cdot \boldsymbol{q}^{*}\right)-\left(\nabla^{*}\right.\right.$. $\left.\left.\boldsymbol{q}^{*}\right)\left.\right|_{t^{*}=0}\right] /\left.e^{*}\right|^{-1}$, where $\left.\left(\nabla^{*} \cdot \boldsymbol{q}^{*}\right)\right|_{t^{*}=0}$ is the divergence of the heat flux at time zero. We investigate the ratio $\Xi^{*} / t_{\mathrm{th}}^{*}$ in order to assess the role of heat conduction in the dynamics of the four experiments. We obtain average values of 500 (B2), 1000 (B1), 2200 (A2), and 4500 (A1) respectively, showing that the heat conduction is of minor importance. There are, however, a few locations in space where the above ratio becomes of the order of unity. This happens in the vicinity of the ad hoc diffusion region, at the bow shocks of the reconnection jets, and at some changing positions along the slow MHD shocks. In the former region the changes are dominated by the heat conduction, while in the latter two dominant is the ad-hoc background heating, $\left.\left(\nabla^{*} \cdot \boldsymbol{q}^{*}\right)\right|_{t^{*}=0}$.
From this simple analysis on the dynamical time-scales of radiation and anisotropic heat conduction, it appears that these effects are only important in a few localized regions of the domain. The ad-hoc background heating is similarly important, as it dominates the development of the thermal energy in a few other localized regions. The time-scales on which changes in the thermal energy takes place are, however, significantly longer than those involved in the dynamical evolution of the reconnection jets. In conclusion, the overall role of radiation, heat conduction and background heating is of minor importance in the timedependent evolution of the reconnection events presented here.

\subsection{Experiments " $A$ "}

As one would expect, the reconnection jet propagating towards the high temperature region ("blue" jet hereafter) appears at higher outflow velocity than the one moving towards the high density region ("red" jet hereafter). The "blue" jets in experiments "A" expand into a region with decreasing mass density and increasing temperature, while 

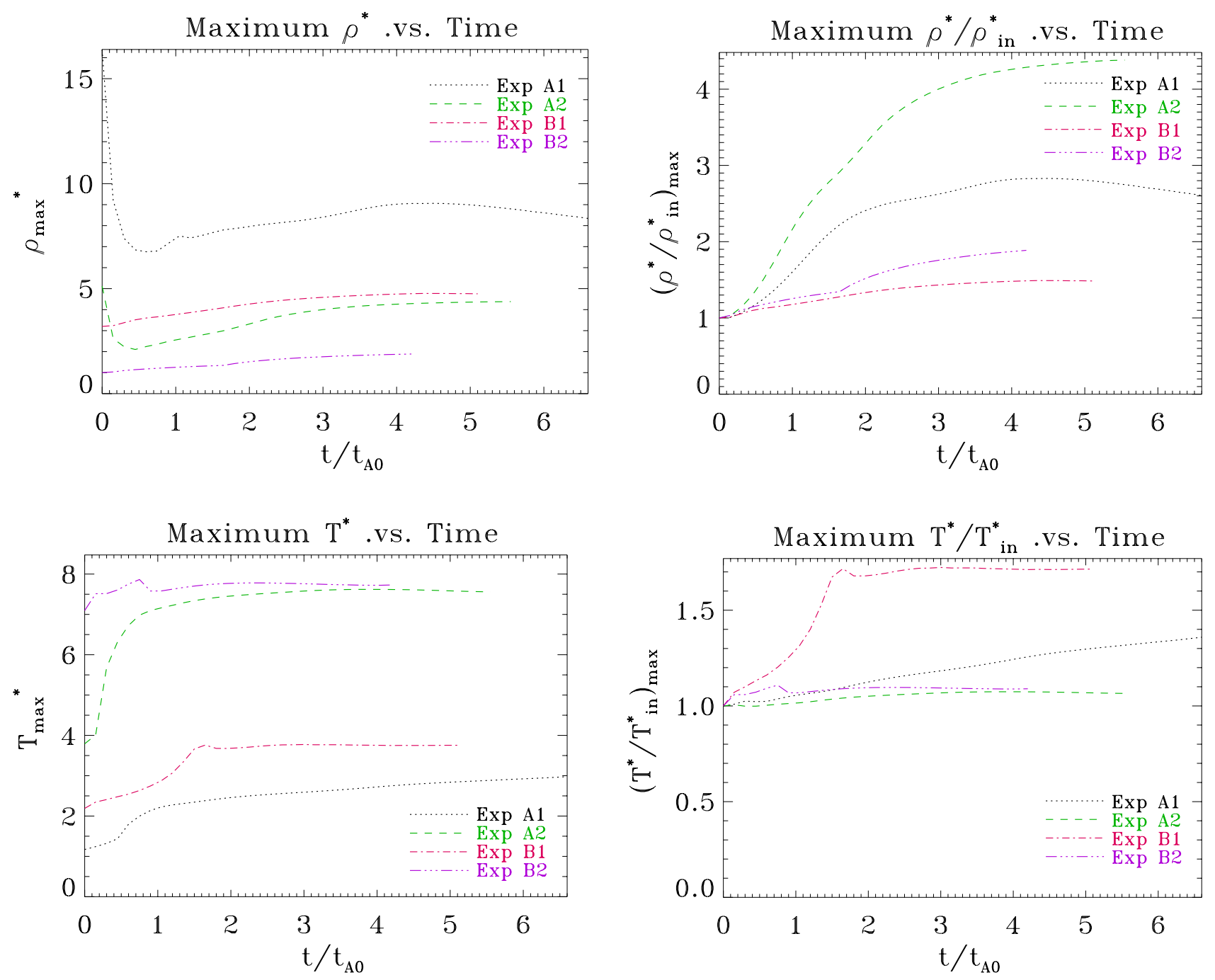

Fig. 10. Set of plots showing the maximum values of various physical quantities along the symmetry axis $x^{*}=0$ (see text for details).

for the "red" counterparts the physical situation is just the opposite. Since the Lorentz forces involved in the acceleration of the jet plasma are of the same magnitude, the acceleration of the "blue" jets is naturally larger than for the "red" ones.

The two "blue" jets seen in Fig. 5 (right column) reveal a distinct "tulip-like" shape. Inside the jet core there is a relatively "cold" region notable in the adjacent image plots. From the density images shown in Fig. 7, it becomes clear these cold regions consist of dense plasma. The highest outflow velocities of the two "blue" jets are reached along the symmetry axis $x^{*}=0$, and inside these dense and relatively cold jet cores. An analysis of the "blue" jets along this symmetry axis was made, and the results are shown in Fig. 9. In the upper left panel of this figure the maximum values of the jet velocity, $V_{\text {jet }}^{\max }$, are plotted as a function of time. The locations at which the peak value of the jet velocity occurs were also derived, and in the top right panel of this figure is shown $V_{\text {jet }}^{\max }$ versus its location.

The mass density, $\rho_{\text {jet }}^{*}$, at the location of peak jet velocity as a function of time is shown in the lower left panel of Fig. 9, while the corresponding temperature, $T_{\text {jet }}^{*}$, of the jet as a function of time is given in the lower right panel. For a comparison, Fig. 10 shows the maximum values of the mass density, $\rho_{\max }^{*}$ (top left), and temperature, $T_{\max }^{*}$ (bottom left) as a function of time reached along the symmetry axis $x^{*}=0$ in the four experiments. This figure also shows the maximum values of $\left(\rho^{*} / \rho_{\text {in }}^{*}\right)_{\max }$ (top right), and $\left(T^{*} / T_{\text {in }}^{*}\right)_{\max }$ (bottom right) as a function of time, respectively. The plots enable one to imagine the observable consequences of these jets in the emitted spectra (choosing as a line-of-sight the symmetry axis $x^{*}=0$ ).

One important result is clear. After a certain time the high velocity regions of the "blue" jets do not undergo significant changes in temperature. As they are relatively denser than the surrounding material, it is very likely that these jets will appear in the spectra of resonant transition region lines as strongly blue-shifted components. As shown in Paper 1 and based on experiment "A1", these "blue" jets indeed appear as strongly blue-shifted Doppler components in the line profiles of C IV (1548.2 $\AA)$ and $\mathrm{O}$ VI $(1031.9 \AA)$ resonant lines, and could be 
interpreted as explosive events. A detailed comparison between the observable consequences of the jets obtained from experiments "A" and "B", such as line synthesis in resonant transition region lines, is presented in Roussev et al. (2001b).

Since the mass density in the current concentration relevant to experiment "A1" is higher than the one in experiment "A2", the "blue" jet in "A2" evolves on a shorter dynamical time-scale than the "blue" jet in "A1". This is clearly visible in Fig. 9 (top row). In both experiments, the "blue" jet outflow velocity approaches the Alfvén velocity of the external region, $V_{A 0}$, in later stages of their evolution (note that this first occurs in experiment "A2").

The dynamical changes of $\rho_{\text {jet }}^{*}$ are largest in experiment "A1", while $T_{\mathrm{jet}}^{*}$ for this experiment is smoother than in experiment "A2" (note also that the initial temperature of the current concentration for experiment "A2" is higher than in "A1"). As to $\rho_{\max }^{*}$ in experiment "A1", the largest changes occur at the early stage of the jet evolution, while remaining weakly dependent on time at the later stages. The biggest differences are observable in $\left(\rho^{*} / \rho_{\text {in }}^{*}\right)_{\max }$, as the "blue" jets evolve. On the contrary, $\left(T^{*} / T_{\text {in }}^{*}\right)_{\text {max }}$ in both experiments slightly increase in time, while $T_{\max }^{*}$ undergoes more substantial changes, especially for experiment "A2".

\subsection{Experiments " $B$ "}

In the model situations with absence of a transition region, i.e. experiment "B", the physical picture of the magnetic reconnection process is symmetric with respect to both the $x$ - and the $y$-axes. In these two cases, we call "blue" jets hereafter those propagating in the positive $y$-direction, while "red" jets are the ones moving in the negative $y$-direction, respectively. The analysis of the two experiments "B" follows similar logic as the one used to discuss experiments "A" in the previous section.

The temperature and density structures of the outflow jets in these two cases are rather interesting. As seen in Fig. 6, the highest temperature in experiment "B2" (second row) is reached around the "foreheads" of the jets (in front of the region with maximum outflow velocity), while in experiment "B1" (first row of Fig. 6), the hottest parts appear around the region of maximum jet velocity. In contrast, Fig. 8 shows that the highest densities relevant to experiment "B1" are reached in the jets' "foreheads" (first row), whereas in the case of "B2" (second row), the densest parts are found around the region of the maximum jet velocity. Similar to experiments "A", the latter are again observed along the symmetry axis $x^{*}=0$.

The same analysis of the "blue" jets along the symmetry axis, as in the previous section, was made for experiments "B", and the results are shown in Figs. 9, 10. As already mentioned before, since the mass density of the current concentration relevant to experiment "B1" is larger than the one in experiment "B2", the outflow jets in the latter evolve on a shorter dynamic time-scale than the jets in the former. This can be seen in the plot showing the maximum jet velocity versus time (see Fig. 9). Note again that the peak jet velocity approaches the external Alfvén velocity towards the end of the experiments.

Similar to experiments "A", the largest dynamical changes in $\rho_{\text {jet }}^{*}$ are found in experiment "B1" rather in "B2". At a later stage of the jet evolution, however, both $T_{\text {jet }}^{*}$ and $\rho_{\text {jet }}^{*}$ have rather smooth behavior and monotonously decrease with time. For comparison, one can also see in Fig. 10 the maximum values of the mass density, $\rho_{\max }^{*}$ (top left), and temperature, $T_{\max }^{*}$ (bottom left) reached along the symmetry axis $x^{*}=0$ for the two experiments "B" as a function of time.

\section{Conclusions}

We examined the response of various physical environments representing the solar atmosphere to a magnetic reconnection event. The results obtained from our 2D MHD modelling are compared, and it is found that the choice of initial state is clearly crucial to such a modelling. We studied the impact of two model parameters to the reconnection jet dynamics, namely, the inhomogeneity of the mass density across the current concentration described with the $\theta$ parameter, and the density (temperature) jump across the transition region defined through the parameter $\Delta \rho$. As to the former, it was found that the lower the mass density in the current concentration (low value of $\theta$ ), the faster the evolution of the outflow jets. As a result, the reconnection jets of experiments " $\mathrm{A}(\mathrm{B}) 2$ " evolved on shorter dynamic time-scales than the ones in experiments "A(B)1". Furthermore, the lower the value of $\theta$, the more cusp-like the shape of the bow shocks is, as well as the more confined the structure of the reconnection jets across the current "sheet" becomes.

As to the other model parameter, $\Delta \rho$, note that it defines the temperature and density stratifications along the current "sheet", and thus clearly introduces an asymmetry in the physical circumstances (experiments "A(B)1"), unless equal to zero (experiments "A(B)2"). Such an asymmetry reflects on the reconnection dynamics, and therefore has an impact on the evolution of the outflow jets (one propagating towards the high temperature region, and another one evolving in the high density region). The latter undergo quite complex development, and evolve on different dynamic time scales. Such a physical picture is expected to inevitably influence the emitting properties of the two jets, as shown by Roussev et al. (2001b). The impact of different physical assumptions regarding the initial state to the emitted spectra is different. By performing line synthesis in resonant transition region lines, however, we could in principle conclude what particular physical circumstances best agree with, and physically explain observational signatures of explosive events. Inspite the simplicity of the 2D model situations examined, one should recognize the qualitative essentials of such a modelling.

Recall the choice of initial state in our modelling, note that we constructed the mass density and temperature profiles across the transition region without making 
explicit assumptions regarding the spatial distribution of the volumetric heating sources. Instead, we simply defined the background heating function through the energy equation as derived for the initial state (see Eq. (13)). An alternative way to construct initial equilibrium solutions would be to make realistic assumptions about the background heating rate first, and then derive the corresponding mass density and temperature profiles across the transition region. Since the physical processes responsible for the heating of the solar atmosphere are yet undetermined, there may be pure speculations about the spatial and temporal distribution of the required background heating for such a model. Recent attempts to theoretically fit temperature profile of coronal loops in 1D, based on observations taken by Yohkoh and TRACE, indicate that the heating of these is dominated by their foot-points (see Mackay et al. 2000; Aschwanden et al. 2000). Constructing the initial state will also have an impact on the importance of the thermal conduction and radiative losses to the physics involved in the numerical modelling. These two processes combined together with the background heating could affect the results of the modelling depending on the particular initial setup chosen. This also could be of importance in studies aimed at calculating the contribution of solar explosive events to the coronal heating. We believe that numerous small scale transient events on the quiet Sun, like explosive events, could provide substantial heating of the solar transition region and overlying corona.

As a further step in numerical studies relevant of explosive events, line synthesis in representative transition region lines (i.e. C IV 1548.2 $\AA$ and O VI $1031.9 \AA$ ) based on the MHD results presented here is a necessary issue. This way it becomes possible to directly compare the results of our MHD modelling with observable quantities, such as line profiles. Results of such studies can be found in Roussev et al. (2001b), where there is also a strong emphasis on the departures from the equilibrium ionization. It is concluded the consideration of non-equilibrium ionization is critical and necessary in any study relevant to explosive events (see also Sarro et al. 1999), since these dynamic events involve time scales smaller than the ionization and recombination times.

Acknowledgements. The National Center for Atmospheric Research (NCAR) is sponsored by the National Science Foundation. Research at Armagh Observatory is grant-aided by the N. Ireland Dept. of Culture, Arts and Leisure. This work was in part supported by PPARC grants PPA/G/S/1999/00055 and PPA/V/S/1999/00668. This work was supported by a grant from the British Council - Acciones Integradas Program (Spain) ref. no. 1814. I. Roussev dedicates his studies to Ana and Elena. K. Galsgaard was supported by PPARC in a form of an Advanced Fellowship. R. Erdélyi is grateful to M. Kéray for patient encouragement. R. Erdélyi acknowledges the financial support obtained from the NSF, Hungary (OTKA, ref nr. TO32462). K. Galsgaard and I. Roussev are grateful for the warm hospitality they received during their visits at Space \& Atmosphere Research Center
(SPARC), Department of Applied Mathematics, University of Sheffield, where part of this work was prepared. I. Roussev is also thankful to the Department of Applied Mathematics, University of St. Andrews, and the NSO in Tucson, Arizona, for the support provided during his visits there. The MHD experiments were carried out on the PPARC funded Compaq MHD Cluster in St. Andrews. We are also thankful to Phil Judge and B.C. Low for the useful discussions while this paper was prepared.

\section{References}

Aschwanden, M. J., Nightingale, R. W., \& Alexander, D. 2000, ApJ, 541, 1059

Brueckner, G. E., \& Bartoe, J.-D. F. 1983, ApJ, 272, 329

Chen, P. F., Fang, C., Tang, Y. H., \& Ding, M. D. 1999 ApJ, 513,516

Cook, J. W., Cheng, C.-C., Jacobs, V. L., \& Antiochos, S. K. 1989, ApJ, 338, 1176

Dere, K. P., Bartoe, J.-D. F., \& Brueckner, G. E. 1989, Solar Phys., 123, 41

Erdélyi, R., De Pontieu, B., \& Roussev, I. 2001, Solar Phys., submitted

Erdélyi, R., De Pontieu, B., \& Sarro, L. M. 1999, ESA-SP, 448, 1345

Erdélyi, R., Doyle, J. G., \& Perez, E. P. 1997, ESA-SP, 404, 353

Erdélyi, R., Sarro, L. M., \& Doyle, J. G. 1998, ESA SP, 421, 207

Forbes, T. G., \& Priest, E. R. 1984, Solar Phys., 94, 315

Innes, D. E., Inhester, B., Axford, W. I., \& Wilhelm, K. 1997, Nature, 386, 811

Innes, D. E., \& Tóth, G. 1999, Solar Phys., 185, 127

Jin, S.-P., Inhester, B., \& Innes, D. 1996, Solar Phys., 168, 279

Mackay, D. H., Galsgaard, K., Priest, E. R., \& Foley, C. R. 2000, Solar Phys., 193, 93

Magara, T., Mineshige, S., Yokoyama, T., \& Shibata, K. 1996, ApJ, 466, 1054

Magara, T., \& Shibata, K. 1999, ApJ, 514, 456

Magara, T., Shibata, K., \& Yokoyama, T. 1997, ApJ, 487, 437

Mariska, J. T. 1992, The Solar Transition Region (Cambridge University Press, Cambridge)

Parker, E. N. 1987, Solar Phys., 110, 11

Perez, M. E., Doyle, J. G., \& Erdélyi, R. 1998, ASP. Conf. Ser., 154,666

Perez, M. E., Doyle, J. G., Erdélyi, R., \& Sarro, L. 1999, A\&A, 342,279

Porter, J. G., Moore, R. L., Reichman, E. J., Engvold, O., \& Harvey, K. L. 1987, ApJ, 323, 380

Priest, E. R. \& Forbes, T. G. 1986, JGR, 91, 5579

Roussev, I., Galsgaard, K., Erdélyi, R., \& Doyle, J. G. 2001a, A\&A, 370, 298

Roussev, I., Erdélyi, R., Doyle, J. G., \& Galsgaard, K. 2001b, A\&A, submitted

Sarro, L. M., Erdélyi, R., \& Doyle, J. G., \& Perez, E. P. 1999, A\&A, 351, 721

Spruit, H. C., Title, A. M., \& van Ballegooijen, A. A. 1987, Solar Phys., 110, 115

Takeuchi, A., \& Shibata, K. 2001, ApJ, 546, L73

Teriaca, L., Doyle, J. G., Erdélyi, R., \& Sarro, L. M. 1999, A\&A, 352, L99

Yokoyama, T., \& Shibata, K. 1996, PASJ, 48, 353 